\title{
CDISC SDTM Laboratory Test Standard Character Result Terminology
}

National Cancer Institute

\section{Source}

National Cancer Institute. CDISC SDTM Laboratory Test Standard Character Result

Terminology. NCI Thesaurus. Code C102580.

Terminology associated with the laboratory test standard character result codelist of the Clinical Data Interchange Standards Consortium (CDISC) Study Data Tabulation Model (SDTM). 